\title{
Computed Tomography of the Inguinal Region with Contrast
}

National Cancer Institute

\section{Source}

National Cancer Institute. Computed Tomography of the Inguinal Region with Contrast. NCl Thesaurus. Code C137889.

Computed tomography of the inguinal region with the use of a contrast agent to enhance the image. 